\title{
Magnetization of AGN jets as imposed by leptonic models of luminous blazars
}

\author{
Mateusz Janiak, Marek Sikora and Rafal Moderski \\ Nicolaus Copernicus Astronomical Center, Bartycka 18, 00-716 Warsaw, Poland \\ email: mjaniak@camk.edu.pl
}

\begin{abstract}
Recent measurements of frequency-dependent shift of radio-core locations indicate that the ratio of the magnetic to kinetic energy flux (the $\sigma$ parameter) is of the order of unity. These results are consistent with predictions of magnetically-arrested-disk (MAD) models of a jet formation, but contradict the predictions of leptonic models of $\gamma$-ray production in luminous blazars. We demonstrate this discrepancy by computing the $\gamma$-ray-to-synchrotron luminosity ratio (the $q$ parameter) as a function of a distance from the black hole for different values of $\sigma$ and using both spherical and planar models for broad-line region and dusty torus. We find that it is impossible to reproduce observed $q \gg 1$ for jets with $\sigma \geqslant 1$. This may indicate that blazar radiation is produced in reconnection layers or in spines of magnetically stratified jets.
\end{abstract}

Keywords. quasars: jets, radiation mechanisms: non-thermal, acceleration of particles

\section{Introduction}

Measurements of radio core-shifts in core dominated luminous radio-loud AGN (Pushkarev et al. (2009)) were used by Zamaninasab et al. (2014) to estimate magnetic fluxes in parsec scale jets. The magnetic fluxes were found to be consistent with magnetic fluxes predicted to thread the black hole by the MAD model (McKinney et al. (2012)). These studies indicate also that magnetization of a jet plasma at parsec distances corresponds to $\sigma \sim 1$. While energetics of blazars seems to be consistent with the MAD model, having in the blazar zone $\sigma \sim 1$ was shown by Nalewajko et al. (2014) to contradict with popular, external-Compton-radiation (ERC) models of luminous, $\gamma$-ray dominant blazars. We verify analytical results of Nalewajko et al. (2014) by numerical studies of SEDs of luminous blazars.

\section{The model}

We calculate the quasi-steady-state electron distribution and produced radiation. Our one-zone leptonic model involves a source of non-thermal radiation propagating down the jet with bulk Lorentz factor $\Gamma=15$ and $\theta_{j e t}=1 / \Gamma$. Synchrotron and ERC radiation is $L_{s y n} \propto u_{B}^{\prime}$ and $L_{E R C} \propto u_{e x t}^{\prime}$, respectively, thus

$$
q \propto \frac{u_{e x t}^{\prime}}{u_{B}^{\prime}}=\frac{(1+\sigma) \Gamma^{2} \zeta \eta_{d i s k}}{4 \sigma \eta_{j e t}}
$$

where $u_{B}^{\prime}$ and $u_{e x t}^{\prime}$ are the energy densities of magnetic field and external photons, respectively, $\eta_{d i s k}=L_{d i s k} / \dot{M} c^{2}$ is accretion disk radiative efficiency, $\eta_{\text {jet }}=L_{\text {jet }} / \dot{M} c^{2}$ is jet production efficiency, $\sigma=L_{B} / L_{k i n}$ and $\zeta=4 \pi u_{e x t}^{\prime} r^{2} c / L_{d i s k} \Gamma^{2}$. Sources of external seed photons in ERC process include broad line region (BLR, $0.1 r_{s u b}<r_{B L R}<r_{s u b}$ ), hot dust region (HDR, $r_{\text {sub }}<r_{H D R}<10.0 r_{\text {sub }}$ ) and accretion disk (where $r_{\text {sub }} \approx 1.0 \mathrm{pc}$ ). We consider both planar and quasi-spherical geometries of BLR and HDR. Values of 

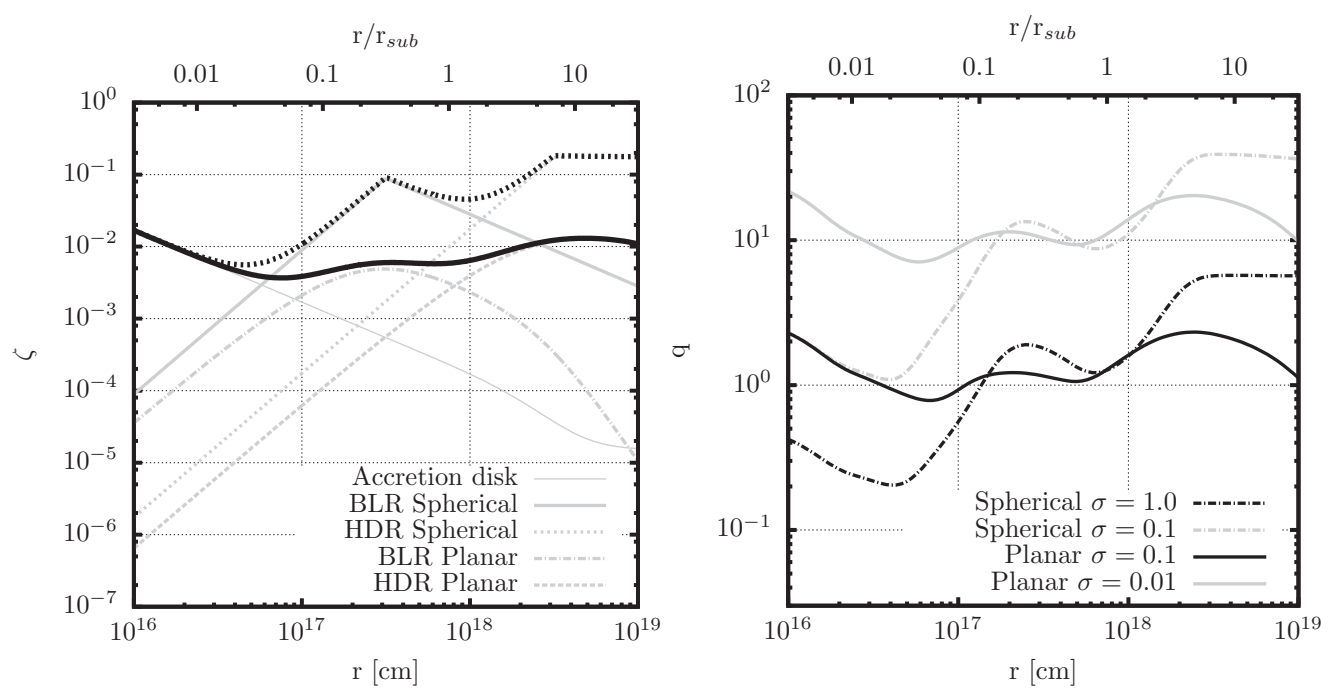

Fig. 1 Left panel: $\zeta$ vs radius for spherical and flat geometries of external radiation sources. Black dotted line is a total $\zeta$ for spherical case and black solid line presents a total $\zeta$ for planar geometry. Right panel: Compton dominance parameter $q$ vs radius for different external source geometries and values of magnetization $\sigma$.

the Compton-dominance parameter $q=L_{E R C} / L_{\text {syn }}$ and $\zeta$ parameter are calculated for three distance decades, $10^{16}-10^{19} \mathrm{~cm}$ and presented in Fig. 1.

\section{Conclusions}

- Modelling of radiation from blazar jets together with the assumption of high Compton dominance leads to $\sigma \ll 1$.

- Calculated values of $u_{e x t}^{\prime}$ in the case of quasi-spherical geometry are much higher than for flat case. But even then $\sigma=1.0$ is too high to reproduce $q>10$ often observed in luminous blazars.

- Possible solution to presented discrepancy between $\sigma \sim 1$ determined by core-shift measurements and much smaller $\sigma$ as indicated by blazar models with $q>>1$ is nonuniformity of magnetic fields across the jet and production of blazar radiation in sites of lower magnetized plasma possibly associated with reconnection layers or with less magnetized jet spines.

\section{Acknowledgements}

We acknowledge financial support by the Polish NCN grant DEC-2100/01/B/ST9/04845.

\section{References}

McKinney, J. C., Tchekhovskoy, A., \& Blandford, R. D., 2012, MNRAS 423, 3083

Nalewajko, K., Begelman, M. C., \& Sikora, M., 2014, ApJ 789, 161

Pushkarev, A. B., Kovalev, Y. Y., Lister, M. L., \& Savolainen, T., 2009, A\& A 507, L33

Sikora, M., Begelman, M. C., \& Rees, M. J., 1994, ApJ 421, 153

Zamaninasab, M., Clausen-Brown, E., Savolainen, T., \& Tchekhovskoy, A., 2014, Nature 510, 126 